\title{
Evaluation of Vitreoretinal Cellular Infiltration in Uveitis on Optical Coherence Tomography
}

Wataru Matsumiya ( $\nabla$ ytkmatsu@med.kobe-u.ac.jp )

Kobe University Hospital

\section{Sentaro Kusuhara}

Kobe University Hospital

\section{Kyong Kim}

Kobe University Hospital

\section{Ryuto Nishisyo}

Kobe University Hospital

\section{Atsuko Katsuyama}

Kobe Kaisei Hospital

Hisanori Imai

Kobe University Hospital

Atsushi Azumi

Kobe Kaisei Hospital

Akihito Uji

Kyoto University Hospital

\section{Makoto Nakamura}

Kobe University Hospital

\section{Noriyuki Sotani}

Kobe University Hospital

\section{Research Article}

Keywords: vitreoretinal cellular infiltration, uveitis, interobserver exact agreement, OCT-based grading system

Posted Date: December 7th, 2020

DOl: https://doi.org/10.21203/rs.3.rs-118504/v1

License: (9) (1) This work is licensed under a Creative Commons Attribution 4.0 International License. Read Full License 


\section{Abstract}

This study aimed to retrospectively evaluate vitreoretinal cellular infiltration in uveitis on spectral domain optical coherence tomography (SD-OCT). Forty eyes of 26 patients with uveitis in the posterior segment and 22 eyes with no apparent abnormalities from 22 individual controls were enrolled. SD-OCT crosssectional images through the fovea in all subjects were graded as follows: grade 0 , absence of cell-like hyperreflective particle (CLHP); grade 1, $\leq 10$ CLHP on the retinal surface without vitreous CLHPs; grade 2, retinal CLHPs with $\leq 5-10$ CLHPs in the vitreous; grade 3, 11-50 vitreous CLHPs; and grade 4, $\geq 50$ vitreous CLHPs with diffuse distribution. The sensitivity and specificity for detecting active intraocular inflammation in the classification with the presence or absence of the positive OCT-based grading scores (grade $\geq 1$ ) were $85 \%$ and $95.5 \%$ respectively. Moreover, the OCT-based grading score was significantly correlated with the vitreous haze classification $(R=0.35, p=0.029)$. The interobserver exact agreement of the OCT-based grading score was moderate $(\mathrm{K}=0.50)$. Thus, this newly developed OCT-based grading system for vitreoretinal cellular infiltration could a potential biomarker to evaluate inflammation in the posterior segment of the eye.

\section{Introduction}

Uveitis can be caused by more than 100 disorders associated with intraocular inflammation [1]. Some have infectious etiologies, and another are caused by systemic disease, including autoimmune diseases, although the causes can be unknown or unidentified in many cases. Uveitis is also well known worldwide as a disease that potentially leads to blindness and accounts for $25 \%$ of cases of total social blindness $[2,3]$. In terms of clinical practice and research, the evaluation of severity and activity objectively in uveitis is an important and challenging issue. Therefore, the classification of uveitis based on the degree of inflammation is crucial for the support of follow-up examinations to evaluate the response to treatment in clinical practice and standardization of evaluation to maintain the quality of clinical research in different sites [4]. In 2005, the SUN working group developed grading schemes for the degree of inflammation in uveitis [5]. These grading schemes for the severity and activity of uveitis have been established in anterior chamber cells, anterior chamber flare, and vitreous haze. However, the SUN working group revealed that no consensus could be reached on the grading system for vitreous cells [5].

Conversely, recent advances in optical coherence tomography (OCT) technology have enabled visualization of intraocular inflammatory cells as hyperreflective particles [6,7]. Moreover, in a mouse model of experimental autoimmune uveoretinitis (EAU), a spectral domain OCT (SD-OCT) scoring system was already used to evaluate the severity of ocular inflammation with intraocular cellular infiltration [8]. By contrast, to the best of our knowledge, there has been no grading to evaluate uveitis. Therefore, we attempted to evaluate vitreoretinal cellular infiltration with SD-OCT and proposed a new OCT-based grading system on vitreous cell infiltration in uveitis.

\section{Results}




\section{Patient characteristics}

In 26 patients with posterior or panuveitis, the mean ages were $55.0 \pm 19.1$ yo with a male:female ratio of 9:17. In 40 eyes with uveitis, 26 (50\%) eyes had granulomatous uveitis, and 14 (27\%) eyes had nongranulomatous uveitis. Six (15\%) eyes exhibited infectious uveitis, and 34 (85\%) eyes demonstrated noninfectious uveitis. In 22 control subjects, the mean ages were $52.3 \pm 17.1$ with a male:female ratio of 10:12.

Table 1 shows the causes of uveitis in the eyes included in this study. The major causes were sarcoidosis $(n=4)$ and Vogt-Koyanagi-Harada disease $(n=4)$. There were 16 eyes with unclassified intraocular inflammation.

\section{Distribution of eyes with ocular inflammation based on the OCT-based grading}

We classified 40 eyes with ocular inflammation in posterior retina into the five categories (grade 0 -grade 4) based on OCT-based grading (Figure 1). Of all 40 eyes, there were 6 eyes in grade 0 as the absence of cell-like hyperreflective particle (CLHP), 7 eyes in grade 1 with several CLHPs ( $\leq 10$ particles) on the retinal surface but without vitreous CLHP, 12 eyes in grade 2 with retinal CLHPs with $\leq 5-10$ CLHPs in the vitreous, 10 eyes in grade 3 with 11-50 vitreous CLHPs and 5 eyes in grade 4 with $\geq 50$ vitreous CLHPs with diffuse distribution..

\section{Sensitivity and specificity of the OCT-based grading}

Table 2 shows the sensitivity, specificity, and interobserver agreement of modalities in the diagnosis of active inflammation. Regarding sensitivity, OCT-based grading (grade $\geq 1$ ) was as good as those of slit lamp examination and fundus photography ( $85 \%$ and $93 \%$, respectively) and better than the presence of vitreous CLHPs on SD-OCT (67.5\%). With respect to specificity, all modalities were good. The grade for vitreous cells by slit lamp examination and fundus photography was $100 \%$, and that for the presence of vitreous CLHPs and OCT-based grading (grade $\geq 1$ ) was 95.5\%. In the interobserver agreement of modalities, the presence or absence of intravitreal CLHPs on SD-OCT and positive scores based on OCTbased grading (grade $\geq 1$ ) were almost perfect ( $k=0.97$ and $k=0.84$, respectively), but those in fundus photography were substantial $(\mathrm{k}=0.75)$.

\section{Reproducibility of OCT-based grading scores}

Table 3 shows the numbers of patients with the exact score classified by OCT-based grading in uveitis and vitreous haze classification. The interobserver agreement of the score in the OCT-based grade was moderate $(\mathrm{K}=0.5)$.

Moreover, the score in the OCT-based grading in uveitis was significantly correlated with the score in the vitreous haze classification $(R=0.345, p=0.029)$, as shown in Figure 2 .

\section{Discussion}


In this study, we showed that SD-OCT could show vitreoretinal cellular infiltration in uveitis as CLHP and proposed a new grading system of the severity of inflammation in uveitis. The sensitivity and specificity were good in the presence of inflammation using the OCT-based grading (grade $\geq 1$ ), and the interobserver agreements were comparable with standard vitreous haze classification. Moreover, this OCT-based grading of uveitis matched a clinical classification in the vitreous haze.

The SUN working group proposed the Grading Scheme for Anterior Chamber Cells and Flare as standard grading scales to evaluate inflammation in the anterior segment [5]. Conversely, a representative grading scale as a vitreous inflammation indicator has been vitreous haze at present, which was a complex indicator because the worsened vitreous haze was caused by the accumulation of some factors: inflammatory cells and proteins in the vitreous [9]. Therefore, when the score of vitreous haze changed, we must consider various factors that could affect it. The recent study of SD-OCT to measure vitreous haze was also reported [10]. By contrast, the SUN working group could not reach acceptance with the classification of inflammatory cells in vitreous.

Recently, as the OCT image resolution improves, some clinical studies reported that SD-OCT could capture even subtle intraocular inflammation [6,7]. Especially in toxoplasmosis, intraretinal hyperreflective particles around active lesions were detected even in time domain OCT. Moreover, OCT showed vitreous hyperreflective particles since SD-OCT was released $[11,12]$. Oréfice et al. showed that scattering hyperreflective particles in the vitreous were observed in 18 eyes of 24 eyes $(75 \%)$ in patients with ocular toxoplasmosis [12].

In practice, Spectralis OCT, which we used as representative SD-OCT in this study, had the optical axial resolutions of $7 \mu \mathrm{m}$ and digital axial resolutions of $3.5 \mu \mathrm{m} /$ pixel. In a mouse model of EAU, SD-OCT matched clinical and pathological findings in any severity of EAU. Previously, fundus photography imagebased grading score had been attempted to evaluate the severity of inflammation in the EAU mouse model. However, OCT-based grading scores via vitreoretinal imaging have been recently established based on its superiority of accuracy in measurement on SD-OCT, compared to fundus photography $[8,13,14]$. Especially, cellular infiltration has been shown to be modified according to the severity of ocular inflammation in EAU. Therefore, the number of cellular infiltrates and other OCT findings could monitor the severity of ocular inflammation. It was shown that choroidal inflammation existed as a high reflectivity of the retinal pigment epithelium when SD-OCT images in EAU model mice met grade $\geq 1$ of EAU.

In this study, classification was based on the spreading area and number of hyperreflective particles according to some previous studies, including the EAU mouse model. Grade 1 indicates a small number of CLHPs and CLHPs limitedly located on the retinal surface. We believe that CLHPs in this stage do not reach the vitreous cavity due to the low severity of inflammation. Grade 2 also indicates a small number of CLHPs, but CLHPs spread to the vitreous cavity because the severity of inflammation in grade 2 is higher than that in grade 1. Grade 3 indicates a number of CLHPs (11-50 particles) more than grade 2. 
Grade 4 indicates a large number of CLHPs and diffuse spread in the vitreous cavity because of the higher severity of inflammation than any other grade.

Reproducibility is important to establish a standard grading system. The SUN working grading score was already evaluated with respect to the interobserver agreement. Kempen et al. reported that exact agreement by three uveitis specialists on the grading of AC cells was 0.34 to 0.43 (low to moderate agreement), that on the grading of $\mathrm{AC}$ flare ranged from 0.50 to 0.64 (moderate agreement), and that grading of vitreous haze was 0.5 (moderate agreement). Moreover, the agreement range was 0.48 to 0.51 (moderate agreement) even when vitreous cells were graded as absent or present [15].

Regarding the interobserver agreement of modalities in this study, the presence or absence of intravitreal CLHPs on SD-OCT and that of positive scores based on OCT-based grading (grade $\geq 1$ ) were almost perfect. They have higher reproducibility than fundus photography with a substantial agreement and the presence of vitreous cells by slit lamp examinations. Moreover, the exact agreement $(\mathrm{K}=0.5)$ of the OCTbased grading score was almost equal to those of any SUN grading score. Therefore, the OCT-based grading system could be acceptable in terms of reproducibility.

The present study had limitations in the study design due to its retrospective nature and a relatively small number of subjects. A prospective study with a larger sample size will be required to confirm the results of the present study. An additional limitation was that only Japanese patients were included and only Spectralis OCT was used in OCT imaging. However, our results suggested that SD-OCT could depict vitreoretinal cellular infiltration in uveitis as CLHP and provide a new grading system of the severity of inflammation in uveitis with good sensitivity and specificity to differentiate uveitis in the posterior segment and control subjects. This information might be essential to establish a new grading system using SD-OCT with a different evaluation concept from the SUN working scheme.

To conclude, this newly proposed OCT-based grading in vitreoretinal cellular infiltration can provide good sensitivity and specificity to differentiate uveitis involving the posterior segment and no ocular inflammation subjects and may be applicable in evaluating the severity of ocular inflammation.

\section{Materials And Methods}

\section{Study design and participants}

This is a retrospective comparative chart review focusing on vitreoretinal cellular infiltration using OCT. All cases in this study were Japanese individuals recruited from the Department of Ophthalmology at the Kobe University Hospital in Japan. This study was approved by the Institutional Review Board at the Kobe University Graduate School of Medicine and conducted according to the Declaration of Helsinki. All subjects provided written informed consent.

The patients in this study initially visited the Kobe University Hospital between January 2016 and January 2017. Forty eyes of 26 patients with posterior uveitis or panuveitis and 22 eyes with no apparent 
abnormal ocular findings from 22 individuals as sex-and age-matched controls during the same periods were included in the study. Patients with anterior or intermediate uveitis and cataract grade $\geq 3$ in the Emery-Little classification and without available good-quality OCT images were excluded.

All eyes underwent slit lamp biomicroscopy, SD OCT (Spectralis; Heidelberg Engineering, Heidelberg, Germany), and fundus photography (Topcon TRC50DX, Topcon Inc, Tokyo, Japan).

Fundus photographs that were obtained with $50^{\circ}$ centered on the fovea were also collected. Fluorescein angiography and/or indocyanine angiography were performed as required.

All clinical data for this study were collected from clinical records, which included imaging at the phase with active inflammation before starting a new treatment or additional treatment in the Kobe University Hospital. According to vitreous haze grading, which was based on the scale developed by Nussenblatt et al. and subsequently defined by the SUN working group [5], we also collected a vitreous haze score based on slit lamp examination or fundus photography from the clinical records.

\section{Classification}

In the evaluation of vitreal cellular infiltration, we selected vertical and horizontal cross-sectional SD OCT images through the fovea using B-scan averaging $>50$ images at the first visit at the Kobe University Hospital. Next, a vertical or horizontal scan, on which a larger number of cells were found, was analyzed to evaluate vitreal cellular infiltration. Then, we evaluated the OCT-based grading (Figure 1). The SD OCT cross-sectional images through the fovea in all subjects were graded as follows: grade 0 , absence of CLHP; grade 1, $\leq 10$ CLHP on the retinal surface without vitreous CLHPs; grade 2, retinal CLHPs with $\leq 5-$ 10 CLHPs in the vitreous; grade 3,11-50 vitreous CLHPs; and grade 4, $\geq 50$ vitreous CLHPs with diffuse distribution.

Subsequently, we selected the scan with a higher OCT-based grade score for statistical analyses. If horizontal and vertical scores were equal, we selected the vertical scan. W.M. and S.K. evaluated the OCT grading and the presence/absence of any abnormality on fundus photographs.

\section{Outcomes}

The primary outcome measures were the sensitivity and specificity of the OCT-based classification for detecting active intraocular inflammation. The sensitivity was calculated as the number of uveitis cases that were correctly classified with the presence of vitreous CLHPs on SD OCT image or that of positive scores based on OCT-based grading (grade $\geq 1$ ), divided by all uveitis cases. Specificity was calculated as the number of control cases that were correctly classified, divided by all control cases.

The secondary outcome measures were the correlation of the OCT-based grading score with vitreous haze grading score and interobserver exact agreement of the OCT-based grading scores.

\section{Statistics analyses}


Interobserver agreement was evaluated using the $\mathrm{k}$ method. Using Spearman's coefficient of rank correlation, bivariate correlations were analyzed. Statistical significance was defined as a P-value of $<0.05$. All statistical analyses were conducted using MedCalc version 15.4 software (MedCalc Software, Mariakerke, Belgium), and a P-value of $<0.05$ was considered statistically significant. According to the descriptive system proposed by Landis and Koch, values of $0.0-0.20,0.21-0.40,0.41-0.60,0.61-0.80$, and $0.81-1.0$ correspond to slight, fair, moderate, substantial, and almost perfect agreement, respectively [16].

\section{Declarations}

\section{Author contributions}

W.M., S.K., and A.U. designed the concept of this study.

W.M., K.K. and R.N. collected the data.

W.M. and S.K. conducted statistical analysis.

W.M., S.K., A.U and M.N. interpreted the data.

W.M. and S.K. drafted the work.

W.M., S.K., N.S., K.K., R.N., A.K., H.I. A.A., A.U., and M.N. revised it critically for important intellectual content.

W.M., S.K., N.S., K.K., R.N., A.K., H.I. A.A., A.U., and M.N. approved of the version submitted for publication. All authors agreed to be accountable for all aspects of the work in ensuring that questions related to the accuracy or integrity of any part of the work are appropriately investigated and resolved.

\section{Competing interests}

A.U.'s work has been funded by Topcon, Nidek, Canon and Santen.

All other authors declare no competing interests.

\section{Data availability}

The datasets generated or analyzed during the current study are available from the corresponding author upon reasonable request.

\section{References}

1. Zierhut, M., Deuter, C. \& Murray, P. I. Classification of Uveitis - Current Guidelines (2007). https://doi.org/10.17925/EOR.2007.00.00.77 
2. Rothova, A., Suttorp-van Schulten, M. S., Frits Treffers, W. \& Kijlstra, A. Causes and frequency of blindness in patients with intraocular inflammatory disease. Br. J. Ophthalmol.80, 332-336 (1996). https://doi.org/10.1136/bjo.80.4.332, Pubmed:8703885.

3. Durrani, O. M., Meads, C. A. \& Murray, P. I. Uveitis: a potentially blinding disease. Ophthalmologica218, 223-236 (2004). https://doi.org/10.1159/000078612, Pubmed:15258410.

4. Bloch-Michel, E. \& Nussenblatt, R. B.. International Uveitis Study Group. International Uveitis Study Group recommendations for the evaluation of intraocular inflammatory disease. Am. J. Ophthalmol.103, 234-235 (1987). https://doi.org/10.1016/s0002-9394(14)74235-7, Pubmed:3812627.

5. Jabs, D. A., Nussenblatt, R. B., Rosenbaum, J. T. \& Standardization of Uveitis Nomenclature (SUN) Working Group. standardization of uveitis nomenclature for reporting clinical data. Results of the First International Workshop. Results of the first international workshop. Am. J. Ophthalmol.140, 509-516 (2005). https://doi.org/10.1016/j.ajo.2005.03.057, Pubmed:16196117.

6. Gallagher, M. J. et al. Br. J. Ophthalmol.12, 1680-1685 (2007).

7. Saito, M., Barbazetto, I. A. \& Spaide, R. F.. Intravitreal cellular infiltrate imaged as punctate spots by spectral-domain optical coherence tomography in eyes with posterior segment inflammatory disease. Retina33, 559-565 (2013). https://doi.org/10.1097/IAE.0b013e31826710ea, Pubmed:23042101.

8. Harimoto K, Ito M, Karasawa Y, Sakurai Y, \& Takeuchi M. Evaluation of mouse experimental autoimmune uveoretinitis by spectral domain optical coherence tomography. Br. J. Ophthalmol.98, 808-812 (2014). https://doi.org/10.1136/bjophthalmol-2013-304421, Pubmed:24574437.

9. Kimura, S. J., Thygeson, P. \& Hogan, M. J. Signs and symptoms of uveitis. II. Classification of the posterior manifestations of uveitis. Am. J. Ophthalmol.47, 171-176 (1959). https://doi.org/10.1016/s0002-9394(14)78240-6, Pubmed:13649856.

10. Keane, P. A. et al. Objective measurement of vitreous inflammation using optical coherence tomography. Ophthalmology121, 1706-1714 (2014). https://doi.org/10.1016/j.ophtha.2014.03.006, Pubmed:24835759.

11. Oréfice, J. L. et al. Vitreoretinal morphology in active ocular toxoplasmosis: a prospective study by optical coherence tomography. Br. J. Ophthalmol.91, 773-780 (2007). https://doi.org/10.1136/bjo.2006.108068, Pubmed:17135336.

12. Oréfice, J. L. et al. Spectral optical coherence tomography findings in patients with ocular toxoplasmosis and active satellite lesions (MINAS Report 1). Acta Ophthalmol.91, e41-e47 (2013). https://doi.org/10.1111/j.1755-3768.2012.02531.x, Pubmed:22970804.

13. Xu, H. et al. A clinical grading system for retinal inflammation in the chronic model of experimental autoimmune uveoretinitis using digital fundus images. Exp. Eye Res.87, 319- 326 (2008). https://doi.org/10.1016/j.exer.2008.06.012, Pubmed:18634784.

14. Copland, D. A. et al. The clinical time-course of experimental autoimmune uveoretinitis using topical endoscopic fundal imaging with histologic and cellular infiltrate correlation. Invest. Ophthalmol. Vis. 
Sci.49, 5458-5465 (2008). https://doi.org/10.1167/iovs.08-2348, Pubmed:18757507.

15. Kempen, J. H., Ganesh, S. K., Sangwan, V. S. \& Rathinam, S. R. Interobserver agreement in grading activity and site of inflammation in eyes of patients with uveitis. Am. J. Ophthalmol.146, 813-8.e1 (2008). https://doi.org/10.1016/j.ajo.2008.06.004, Pubmed:18687418.

16. Landis, J. R. \& Koch, G. G. The measurement of observer agreement for categorical data. Biometrics33, 159 -174 (1977). https://doi.org/10.2307/2529310, Pubmed:843571.

\section{Tables}

Table 1. Causes of uveitis

\begin{tabular}{|ll|}
\hline Diagnosis & Number of eyes \\
\hline Sarcoidosis & $4(10.0 \%)$ \\
\hline Vogt-Koyanagi-Harada disease & $4(10.0 \%)$ \\
\hline Scleritis & $3(7.5 \%)$ \\
\hline Cytomegalovirus retinitis & $3(7.5 \%)$ \\
\hline Uveitis with psoriasis & $2(5.0 \%)$ \\
\hline Choroiditis & $1(2.5 \%)$ \\
\hline Acute retinal necrosis & $1(2.5 \%)$ \\
\hline Ocular toxocariasis & $1(2.5 \%)$ \\
\hline Others & $5(12.5 \%)$ \\
\hline Unclassified intraocular inflammation & $16(40.0 \%)$ \\
\hline Total & 40 \\
\hline
\end{tabular}


Table 2. Sensitivity and specificity of modalities in the diagnosis of active

\section{inflammation}

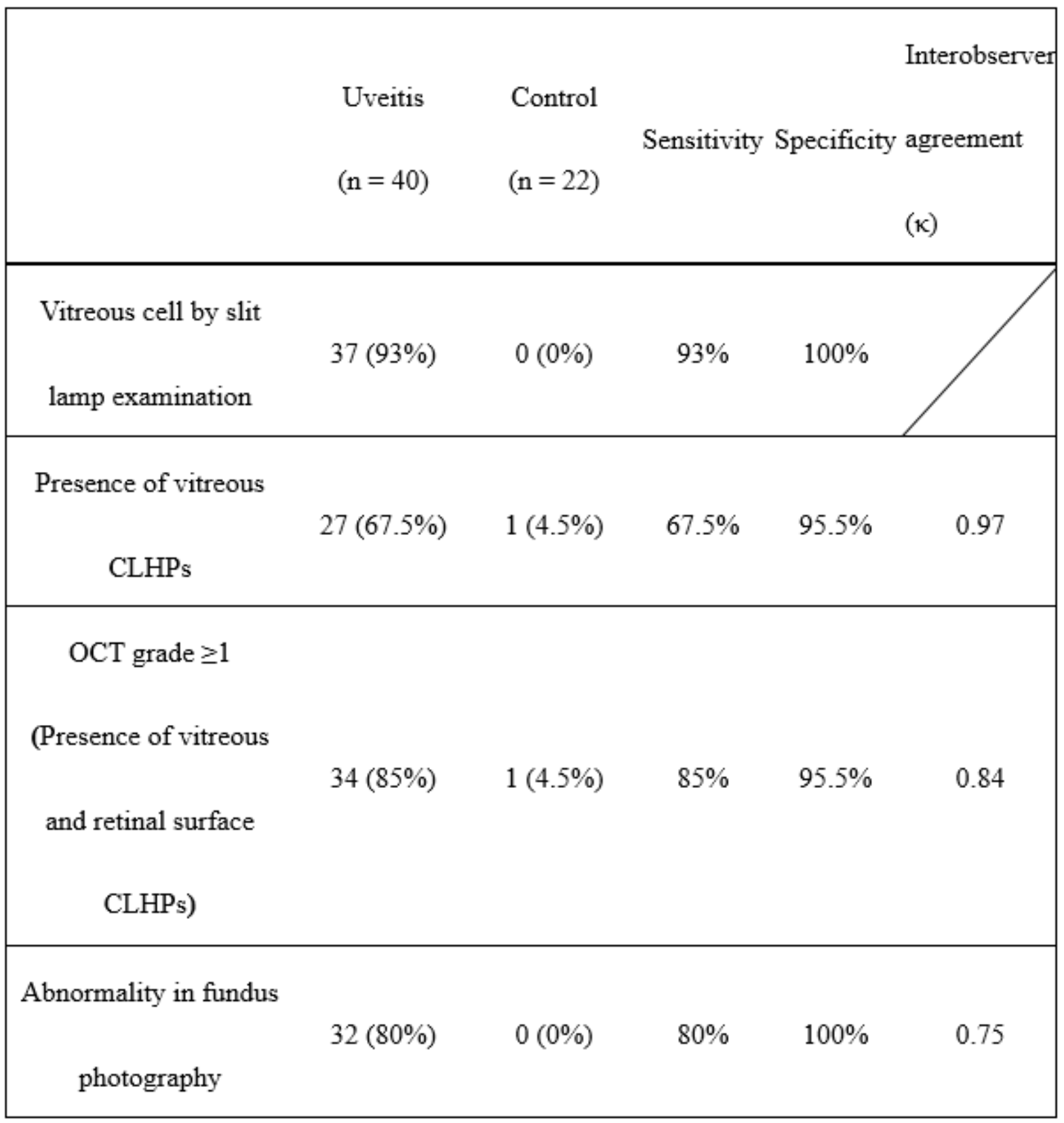


Table 3. OCT-based grade and vitreous haze grade in eyes with uveitis

\begin{tabular}{|c|c|c|c|c|c|c|}
\hline $\begin{array}{c}\text { OCT-based } \\
\text { grade }\end{array}$ & $\mathbf{0}$ & 1 & 2 & 3 & 4 & $\begin{array}{l}\text { Interobserver } \\
\text { agreement } \\
\text { (к) }\end{array}$ \\
\hline Number of eyes & 6 & 7 & 12 & 10 & 5 & 0.50 \\
\hline $\begin{array}{c}\text { Vitreous haze } \\
\text { grade }\end{array}$ & $\mathbf{0}$ & 0.5 & 1 & 2 & 3 & \\
\hline Number of eyes & 6 & 7 & 12 & 10 & 5 & \\
\hline
\end{tabular}

Figures 


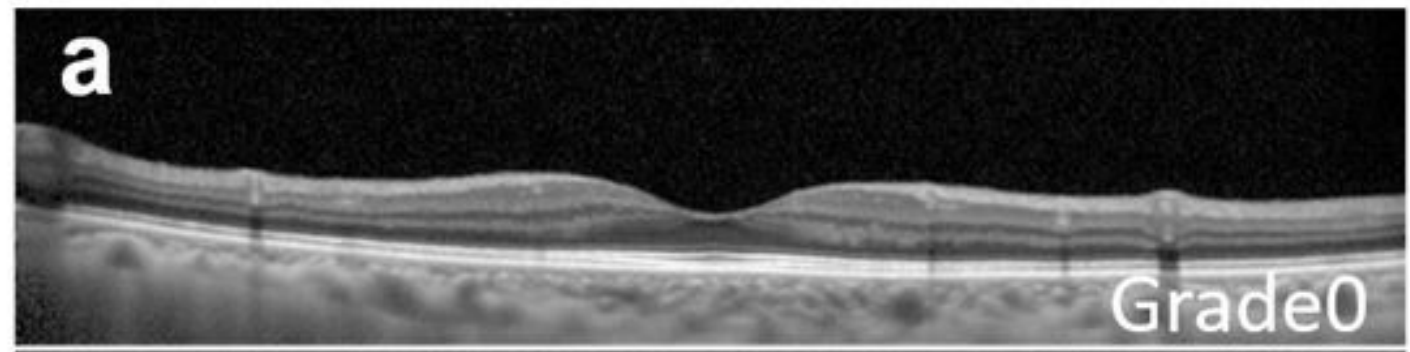

\section{b}
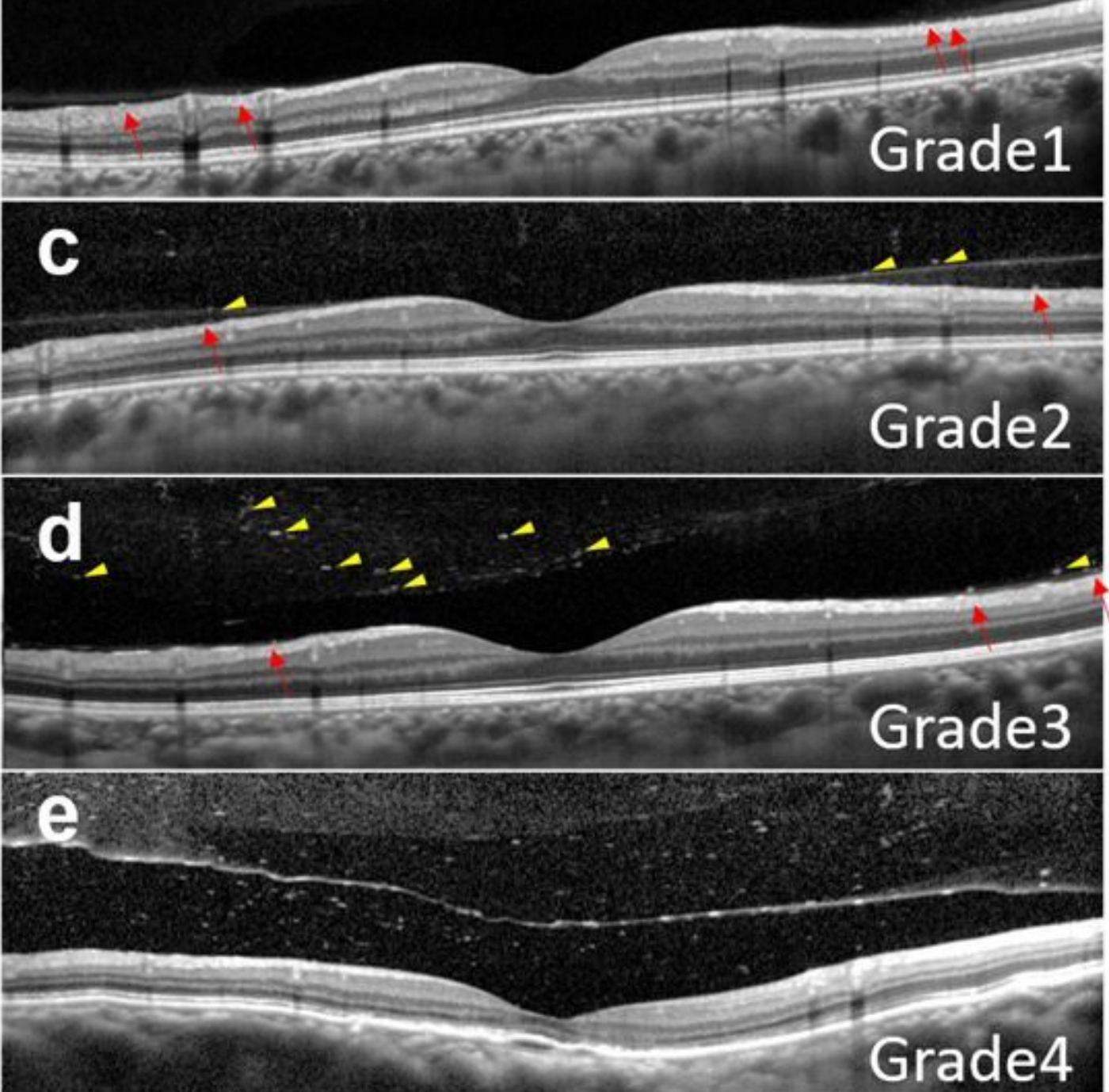

\section{Figure 1}

OCT-based grading in uveitis. CLHP, cell-like hyperreflective particle. (a) Grade 0, absence of CLHP. (b) Grade 1, $\leq 10$ CLHP on the retinal surface without vitreous CLHPs. (c) Grade 2, retinal CLHPs with $\leq 5-10$ CLHPs in the vitreous (d) Grade 3, 11-50 vitreous CLHPs. (e) Grade 4, $\geq 50$ vitreous CLHPs with diffuse distribution. Red arrows show CLHPs on the retinal surface, and yellow arrowheads show CLHPs in the vitreous. 


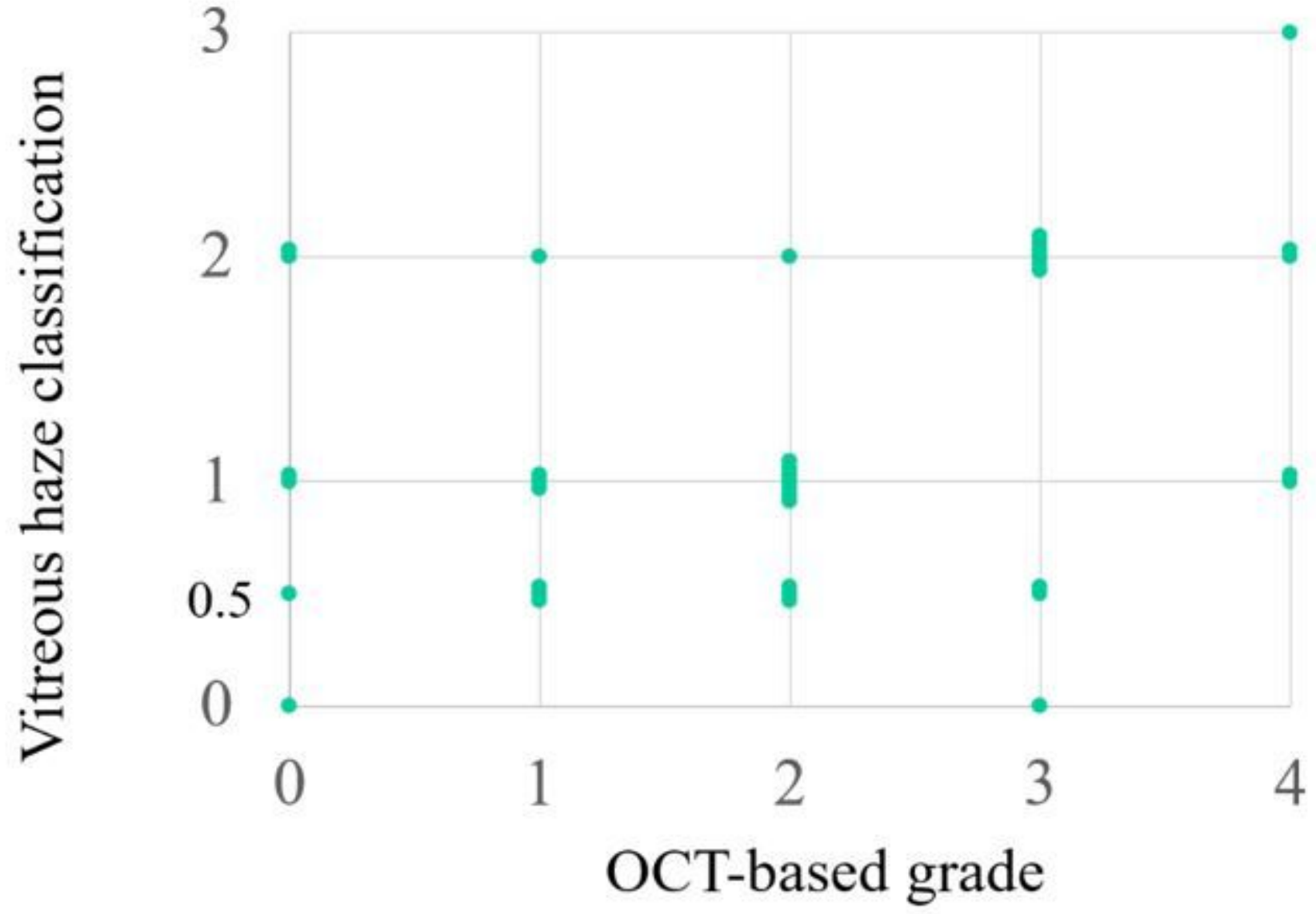

Figure 2

Correlation between OCT-based grade and vitreous haze grade in eyes with uveitis. Spearman's coefficient of rank correlation $(R)$ is 0.345 , and P-value is 0.029 . 